\title{
Retrieval of surface currents from sequential satellite radar images
}

\author{
O. A. Danilicheva, S. A. Ermakov, I. A. Kapustin \\ Institute of Applied Physics RAS, Nizhny Novgorod, 603950, Russia \\ E-mail: olgadan@ipfran.ru
}

\begin{abstract}
Determination of characteristics of marine currents using satellite remote sensing data is a rather complicated problem that has not been completely solved yet. Synthetic aperture radars (SAR) are often used to estimate the velocities of surface currents. The "filamentary" structures associated with biogenic marine films (slicks), which are often observed on the water surface at low-to-moderate wind conditions, can be potentially used as tracers to determine the surface current velocities. In this paper, an attempt is made to characterize marine currents using pairs of sequential images obtained with Envisat ASAR and ERS-2 SAR. The Maximum Cross-Correlation technique has been used to retrieve the surface current field. It is obtained that for some slick structures the retrieved surface velocities are directed nearly along the "filaments", so the latter can be considered as markers of the current streamlines. However, for other slicks, the velocities are directed at rather large angles to the tangents of the "filamentary" structures, so the "filaments" differ from the current streamlines. Supposedly, this is because the currents may not be steady and marine slicks cannot change their orientation and shape instantly following fast changes of environmental conditions, in particular, to variations of wind speed/direction.
\end{abstract}

\section{Introduction}

Remote sensing data is a major source of information about the processes occurring in the surface layer of the ocean. There is a large amount of high-resolution satellite remote sensing data, which are available for free and widely used by researchers. At present, however, the problem of characterization of the ocean upper layer dynamics has not been completely solved. The problem of determination of the field of surface oceanic currents by remote sensing methods is widely discussed in the literature. Information about currents in the microlayer is particularly important in the context of forecasting of pollution transport on the water surface. Marine surface currents can be estimated, for example, when comparing sequential optical or infrared (IR) images [1-3]. However, one of the main disadvantages of optical/IR data is that they are limited by daytime and cloudless conditions. Therefore, synthetic aperture radar is often used to estimate current velocities, because it allows obtaining images with high spatial resolution regardless of weather conditions and time of a day.

It is known that at low-to-moderate wind conditions the "filamentary" slick structures associated with surfactant films appear on the water surface and are easily detected in radar imagery. Very often the "filamentary" slicks are supposed by default as tracers of marine surface currents, so the velocity field of the upper layer presumably can be retrieved using slick structures.

Typically, a pair of sequential radar images of slicks on the water surface is used to estimate the structure displacement. The shift of the structure is monitored using a Maximum Cross-Correlation 
(MCC) method when analyzing the cross-correlation of the sequential images [4-7]. It has been mentioned in $[5,6]$ that slicks indicate the regions of convergence and act as passive tracers and thus can be used for the current field mapping. The question arises, however, how well the shape of the slicks corresponds to the structure of the current field. Recent field experiments [8-11] focused on studies of using artificial film slicks as surface currents and vortexes tracers confirmed the potential possibility of getting information about marine currents from the slick evolution. However, the number of studies where the dynamics of slicks in the surface current field has been investigated is still very limited. Accordingly, the question of how adequately the slicks geometry reflects the field structure of the surface currents and whether it is reasonable to use the slicks as indicators of the surface currents for their quantitative description remains open and requires further analysis.

This paper presents new results of the analysis of several pairs of sequential SAR images with a sufficiently small time interval between the frames (about 30 minutes). The study is aimed to understand better the relationship between the film slicks geometry and the surface current field and to retrieve the currents in the area of interest. The MCC method was used for the current retrieval.

\section{Observation}

Four pairs of consecutive radar images of the water surface with complex film structures were used to analyse the relationship between the shape of the filamentary slick structures and the structures of surface currents. The images were obtained by Envisat ASAR and ERS-2 SAR. One pair was obtained for the southwestern part of the Baltic Sea on 25.04.2009 in the interval from 9:00 to 10:00 UTC, the remaining analysed images were obtained for the western coast of the USA (southwestern California) on 17.04.2008, 28.05.2008 and 08.04.2009 within the interval of 18:00-19:00 UTC. The time interval between successive images for all pairs was about 30 minutes. The spatial resolution of the images is $75 \mathrm{~m}$. The wavelength of both radars is $5.7 \mathrm{~cm}$, and the incidence angle is 23 degrees. The wind was 5-6 m/s according to the QuikScat satellite reflectometer. Indented slick structures, which slightly change their distinctive shape over the time interval between the subsequent frames, are clearly visible in the images. Therefore, it is easy to track the displacement of individual fragments of these structures.

\section{The Maximum Cross Correlation technique}

The Maximum Cross-Correlation Technique (MCC) technique was used to restore the field of surface currents from the displacement of the film structures. This technique is based on dividing the image into many subimages and searching for the maximum of the cross-correlation matrix calculated for each pair of subsequent subimages. The shift of the cross-correlation matrix maximum corresponds to the most probable displacement of the slick structures in the subimage, and the current velocity vectors are calculated using this shift. The size of the subimages determines the spatial resolution of the retrieved current field. Not the full images were analyzed, but their fragments, which include individual slick structures, in order to track the displacement of the slick and its parts. The subimage size was taken of 32 pixels with a step between the centers of adjacent subimages of 16 pixels to obtain additional information about the displacement at the boundaries and angles of each subimage. This information was used to take into account additional shifts and rotations of slick structures in the subimage.

\section{Results and discussion}

The obtained range of the velocity magnitude varies from 0 to $35 \mathrm{~cm} / \mathrm{s}$.

Let us first estimate the accuracy of the obtained velocity vectors. The maximum accuracy of the velocity vector is determined by the displacement of one pixel between the subsequent images. If the shift of a slick structure is less than one pixel, then, obviously, the velocity value is not determined. The error of the velocity vector in our case was $4 \mathrm{~cm} / \mathrm{s}$. Also, there is a geolocation error [4] between the Envisat and ERS-2 images. The estimate of this error was determined by the land displacement between the images and thus obtained error was estimated as $4 \mathrm{~cm} / \mathrm{s}$.

It is clear that an error of the retrieval of velocity magnitude also leads to some uncertainty of the velocity vector direction. For example, for a current magnitude of about $30 \mathrm{~cm} / \mathrm{s}$, the error of direction is about 15 degrees. 
Note that a significant error of the calculating the current velocities using the MCC technique occurs for the cases of displacements of a slick band along itself, particularly if the band has no specific features (or has small-scale features compared to the size of the subimage). That is why the "manual" processing or the technique of the "tracking" of individual slick roughness displacements can be applied along with the software processing using the MCC method [12].

It was shown that the geometry of a part of the slick structures is in good agreement with the structure of the current field, i.e., the slick structures characterize the streamlines of the currents (figure 1 (left)). However, the current velocities of some other parts of the studied regions are directed at a large angle to the slick bands, and the geometry of the slicks is strongly inconsistent with the current streamlines. Supposedly, the reason of this inconsistency is that the currents are nonstationary, and they can change quickly, according to changes of environmental conditions, in particular, of wind magnitude and direction. In this case, slicks cannot change their geometry fast enough, and this leads to an inconsistency between the current streamlines and the slick geometry.
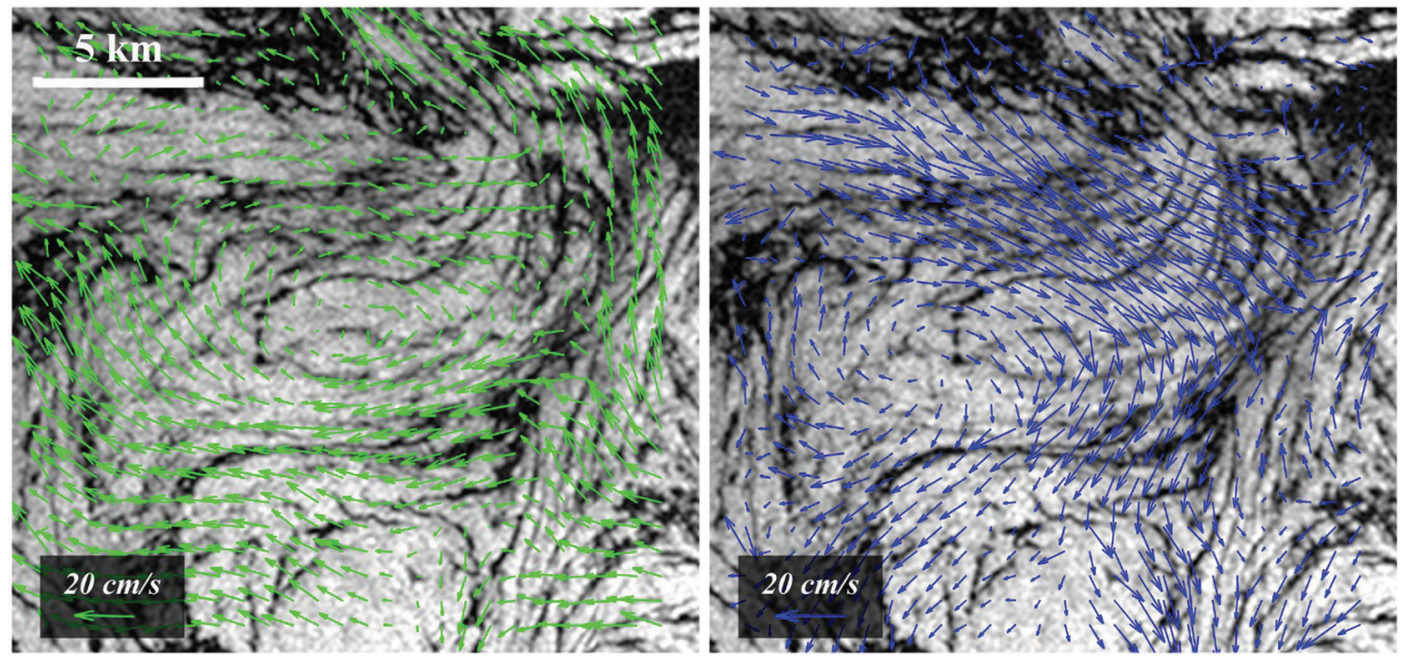

Figure 1. The retrieved current field by the MCC method (left). The current field of currents excluding the wind component (right).

The divergence of current fields retrieved by MCC method was analysed in [13] and also demonstrated a partial correspondence with the slick structure geometry.

It was shown in [8] that the slick band propagation velocity can be described as the sum of the marine current velocity in the upper layer and $3 \%$ of the wind speed. Therefore, if the wind speed is known, the marine current field without taking into account the wind component can be calculated using the retrieved current field by the MCC method. The current field excluding the wind component is shown in figure 1 (right). The vortex structure is seen when analyzing the velocity fields with and without taking into account the wind component. The location of the vortex center is dissimilar in these cases and the distance between the centers about $2.5 \mathrm{~km}$. However, it is difficult to be sure about the reliability of the obtained current fields without taking into account the wind component since the change of the wind over the time between the subsequent radar images was not taken into account and there is no other data of the current field for comparison.

\section{Conclusions}

The surface current fields are retrieved based on the results of processing consecutive satellite radar images of marine slicks. It is demonstrated that the structure of retrieved currents is partly consistent with the geometry of the film structures, and the shape of the slicks marks the streamlines of the currents. However, the field of current velocity vectors may not completely agree with the streamlines, and the velocity vectors can be directed at a sufficiently large angle to the slick bands. Supposedly, this contradiction exists since the surface currents are nonstationary and can change quickly. If the characteristic time of the slick structures restructuring differs significantly from the characteristic time of the current change due to the variations of weather conditions (in particular, the magnitude and direction 
of the wind), then the disagreement between the slick structure geometry and the structure of the current field can be significant.

The current field without taking into account the influence of the wind component is obtained. However, it is difficult to be sure about reliability of the obtained results due to the lack of independent current field measurements for comparison. This shows the need for further research using both remote sensing data and co-located/simultaneous field observations.

\section{Acknowledgements}

This research was funded by the Russian Science Foundation (Project No. 18-77-10066).

\section{References}

[1] Bowen M. M., Emery W.J., Wilkin J.L., Tildesley P.C., Barton I.J., Knewtson R., Extracting multiyear surface currents from sequential thermal imagery using the maximum cross-correlation technique, $J$. Atmospheric and Oceanic Technology, 2002, Vol. 19(10), pp. 1665-1676.

[2] Yang H., Arnone R., Jolliff J., Estimating advective near-surface currents from ocean color satellite images, Remote Sensing Environment, 2015, Vol. 158, pp. 1-14.

[3] Gade M., Fiedler G., Dreschler-Fischer L., Mesoscale Sea Surface Currents Derived from Multi-Sensor Satellite Imagery, ESA Envisat Symp. 2007, Proc., H. Lacoste, L. Ouwehand (eds.), 2007, 466590, 5 p.

[4] Qazi W.A., Emery W. J., Fox-Kemper B., Computing Ocean Surface Currents Over the Coastal California Current System Using 30-Min-Lag Sequential SAR Images, IEEE Trans. Geoscience and Remote Sensing, 2014, Vol. 52(12), pp. 7559-7580.

[5] Lyzenga D. R., Marmorino G. O., Measurement of surface currents using sequential synthetic aperture radar images of slick patterns near the edge of the Gulf Stream, J. Geophysical Research, 1998, Vol. 103(9), pp. $18769-18777$.

[6] Marmorino G. O., Holt B., Molemaker M.J., DiGiacomo P. M., Sletten M.A., Airborne synthetic aperture radar observations of "spiral eddy" slick patterns in the Southern California Bight, J. Geophysical Research, 2010, Vol. 115(C5), C05010. 14 p.

[7] Gade M., Byfield V., Ermakov S., Lavrova O. Mitnik L., Slicks as indicators for marine processes, Oceanography, 2013, Vol. 26(2), pp. 138-149.

[8] Shomina O.V., Ermoshkin A.V., Danilicheva O.A., Tarasova T.V., Kapustin I.A., Ermakov S.A., Slick bands kinematics due to marine current and wind: study and simulation, Remote Sensing of the Ocean, Sea Ice, Coastal Waters, and Large Water Regions, Proc. SPIE, 2019, V. 11150, 111501J. 6 p.

[9] Shomina O. V., Kapustin I.A., Ermoshkin A.V., Ermakov S. A., On the dynamics of articial slick band in the coastal zone of the Black Sea, Sovremennye problemy distantsionnogo zondirovaniya Zemli iz kosmosa, 2019, Vol. 16(4), pp. 222-232.

[10] Kapustin I. A., Shomina O.V., Ermoshkin A. V., Bogatov N. A., Kupaev A. V., Molkov A.A., Ermakov S. A. (2019b), On Capabilities of Tracking Marine Surface Currents Using Artificial Film Slicks, Remote Sensing, 2019, Vol. 11(7), 840. 17 p.

[11] Kapustin I.A., Ermoshkin A.V., Bogatov N.A., Molkov A.A., On the estimation of the contribution of near-surface wind to the kinematics of slicks on the sea surface under conditions of finite wave fetch, Sovremennye problemy distantsionnogo zondirovaniya Zemli iz kosmosa, 2019, Vol. 16(2), pp. 163-172.

[12] Danilicheva O.A., Ermakov S.A., Kapustin I.A., On the retrieval of surface marine currents field using sequential satellite SAR images of slick structures, Sovremennye problemy distantsionnogo zondirovaniya Zemli iz kosmosa, 2020, Vol. 17, pp. 206-214.

[13] Danilicheva O.A., Ermakov S.A., Kapustin I. A., Lavrova O. Y., Characterization of surface currents from subsequent satellite images of organic slicks on the sea surface, Remote Sensing of the Ocean, Sea Ice, Coastal Waters, and Large Water Regions, Proc. SPIE, 2019, V. 11150, 111501R, 7 p. 